\title{
8
}

\section{A combined knowledge and hypermedia system to attain educational objectives}

\author{
Andréa Bender \\ Anneli Edman \\ Lena Sundling \\ Uppsala University \\ Sweden
}

\begin{abstract}
A knowledge system can be a valuable educational tool, however there are four principal problems: lack of control and initiative, difficulties in understanding the reasoning process, absence of overview and problems in comprehending the complexity of the domain. By merging techniques from knowledge and hypermedia systems these problems may be alleviated. This paper presents the functions of such a hybrid system.
\end{abstract}

Main conference themes: artificial intelligence, flexible learning, tutoring

Educational areas: secondary education

Study topics:

Secondary keywords: expert systems, hypermedia, interactive, knowledge based, problem solving, 


\section{BACKGROUND}

'Analyze More' is a knowledge system which aims to support students, mainly in upper secondary school, in their activities in sampling and evaluating the quality of water in lakes. Student should acquire an increased understanding of possible choices in measuring and observation methods, as well as of data interpretation. The development of the system has been done in a cooperation between the National Board of Education, the Swedish Environment Protection Agency, teachers and the Computing Science Department of Uppsala University. The system which is now on sale, is used in about 30 schools (see for further information $[1,2])$.

This 'Analyze More' system was built in Prolog and evaluates the water quality on the basis of a number of indicators which may be divided into the following groups: chemical, physical and biological. Examples of chemical indicators are alkalinity and content of phosphorus, nitrogen, chlorophyll and oxygen. Physical indicators are, for instance, $\mathrm{pH}$-value, conductivity, colour and water transparency. The biological indicators are: presence of plants and animals, both in and around the lake. From the measurements and observations made by the students the system is able to conclude if the lake is oligotrophic, normal or eutrophic. Furthermore the system is able to indicate whether the water in the lake is acid.

From experiences in the Guidon-project [3], amongst others, it is known that special considerations must be taken into account when designing a knowledge system for educational purposes. Both the Knowledge Base (KB) and the user interface of 'Analyze More' have special features so as to facilitate tutoring. Still problems remain in that the system does not contribute to the understanding of the domain to the extent expected.

\section{PROBLEMS AND AN APPROACH TO THEIR SOLUTION}

The four major problems in understanding the domain are: First, the system does not enable the user to maintain control and initiative. Second, the user has severe difficulties in understanding the system's reasoning both on a detailed level of rules and on a higher strategic level. Third, a combination of the two first factors limits the user in acquiring an overview and thereby an understanding of the problem solving process. Finally it is a problem to understand the ecological system as a whole, an understanding strongly emphasized by teachers. Students frequently make the error of solely regarding part of the ecological system without taking into account effects on and correlations with other parts. 
These problems can be tackled in several different ways; our approach is based on extending 'Analyze More' with techniques both from hypermedia and knowledge systems. This combination should be fruitful since it builds on each system's advantages. The fundamental power of knowledge systems lies in their capacity to analyze and solve problems. Unfortunately human beings have considerable difficulty in understanding the system's reasoning process. Hypermedia systems on the other hand offer vast possibilities for obtaining information on individual preferences [4], but lack deductive power. In addition metaknowledge can be incorporated in the knowledge system to improve the successful explanation of the system's reasoning behaviour.

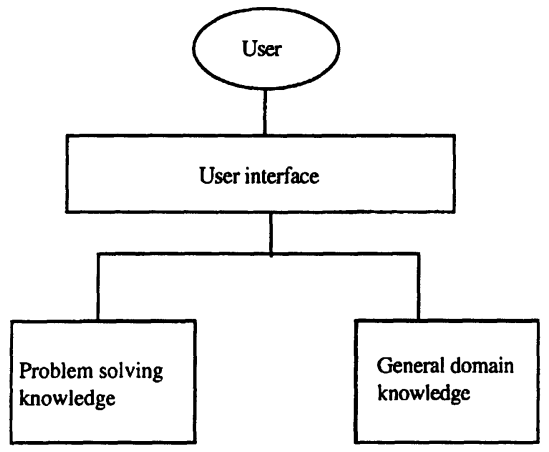

Fig. 1 Two types of knowledge conveyed by the system

The combination of hypermedia and knowledge system supports two types of knowledge we wish to convey, see Figure 1. Problem solving knowledge demonstrates steps which need to be taken for solving a complex problem, otherwise solved by experts in the field. Besides problem solving skills an expert also needs vast general domain knowledge. Parts of this knowledge are not explicitly used, but contribute to the global understanding of the topic. The nature of this knowledge demands a flexible representation which may be best supported by hypermedia. On the other hand problem solving knowledge is preferably represented in a knowledge system.

\section{FACILITIES OFFERED BY THE SYSTEM}

On initial contact with the system a short introduction is given and then a menu appears by which the student can select a topic, see Figure 2. Students may at this time obtain information to help them in selecting a topic. Information on the 
importance of the topic for example. But also the system may be asked to select the order in which to process the topics.

Then a form appears which the user will have to fill in. Each form contains indicators affecting the quality of the water in lakes. A form may contain several indicators which are related to each other. We call such a group of indicators a parameter. Some questions are basic in nature and will always appear while others in contrast depend upon answers previously given. Answers given may also lead to disregarding questions. The form also presents information describing how the indicators affect the water quality.

In interaction with the form additional information may be required by the user. This general domain knowledge may be obtained through the 'Tell $\mathrm{Me}$ More' facility. As can be seen in Figure 2 there are four different types of this: Methods for Measurement and Observation, Information Concerning Indicators, Did you know that ... and Miscellaneous Domain Knowledge. 'Methods for Measurement and Observation' gives detailed information on the practical steps in measurement; for example on how to measure the oxygen content in a lake. The information on indicators consists of:

- definitions;

- intervals (in the case of oxygen content, the value must be between $0-20$ );

- units of measurement (oxygen content is measured in $\mathrm{mg} / \mathrm{l}$ );

- standard margin of errors (here $5 \%$ ).

The 'Did you know that ...' category contains various interesting comments peripheral to the domain. These comments have been included because additional redundant information is necessary in order to facilitate recall from memory through additional paths of association [5]. The 'Miscellaneous Domain Knowledge' offers extra information. Additionally, the 'Why' button supplies information on the relation of one indicator with another and their mutual effects. If needed all the rules in which an indicator or a parameter occurs may be presented.

The user iterates between the menu of topics and the form selecting topics and answering the corresponding questions. When data input is completed, the system can take control, start the deduction process and present its conclusion ('Results' box in Fig. 2). The conclusion is based upon several intermediate results being the product of a group of rules with as common denominator the investigation of the consequences of a certain topic, e.g. algae. The user has the option of obtaining more information about the results: a so-called 'Howexplanation' may be obtained, text describing the general procedure for determining the conclusion. 


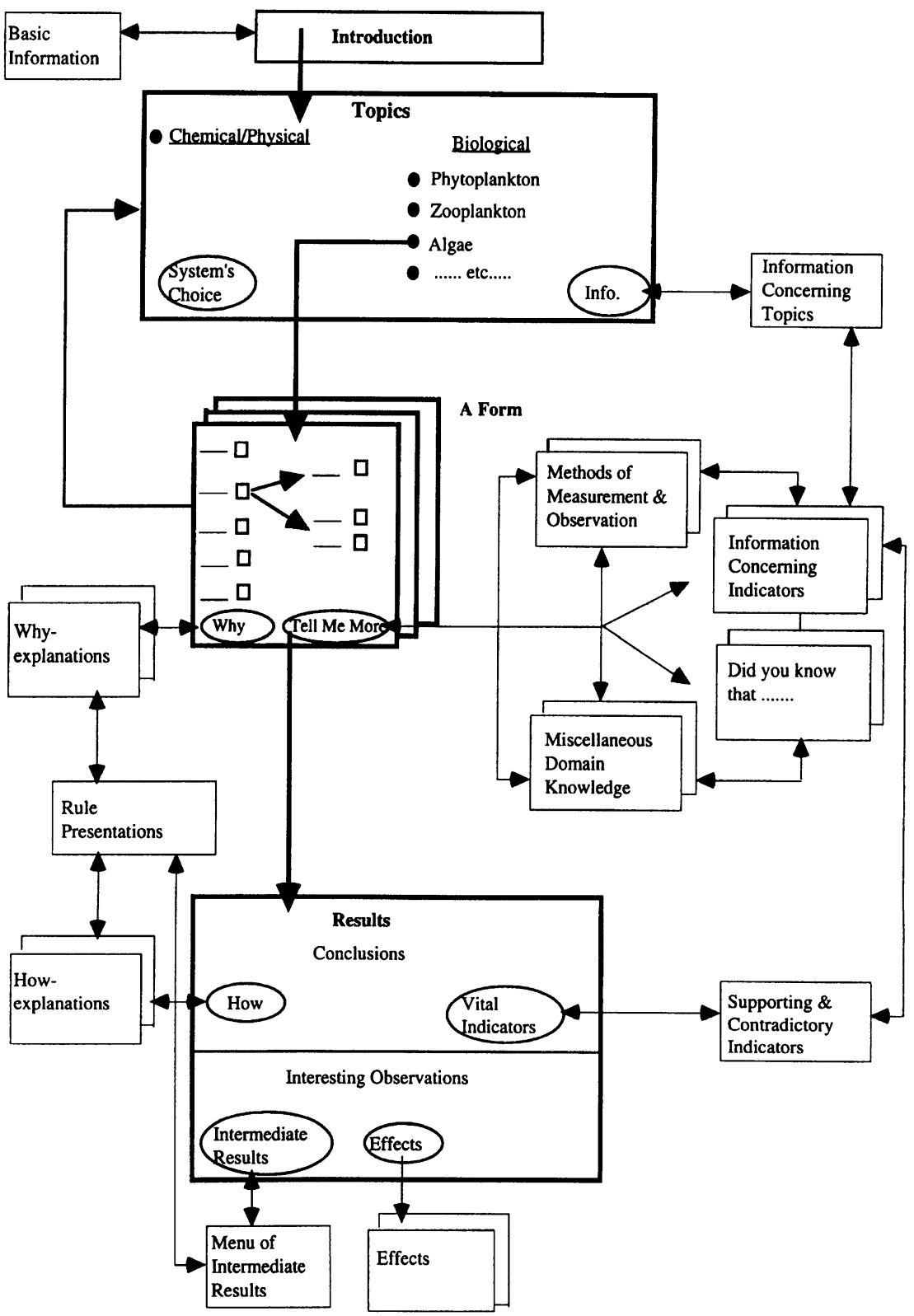

Fig. 2 Functions offered by the system 
The user may proceed to view a list of rules pertinent to the current result. The supporting and contradictory indicators may be examined. To do this the user must choose 'Vital Indicators'. By selecting the 'Intermediate Results' button the user may access more detailed information on the result. This information is structured in the same way as the topic presentation menu. The user may also ask for the rules supporting this information. Finally the user may also press the 'Effects' button which supplies, among other, information concerning long term effects and relations between indicators.

\section{DISCUSSION}

\section{Do the functions of the system offer solutions?}

How do the functions presented above alleviate the problems with control and initiative, with understanding of the system's reasoning, with overview and understanding of the domain? The initial menu oriented presentation offers the possibility of studying topics in an individually desired order which naturally supports the concept of mixed initiative stressed by Shneiderman [6], and also a student is presented with an overview of the central topics pertaining to the domain thereby acquiring vital comprehension of the main issues for solving the problem at hand. Study of the importance of a topic compared to another also contributes to global understanding of the domain. In addition forms permit the user to obtain an overview over the questions on a certain topic and facilitate understanding of the relations between indicators.

The 'Tell Me More' facility offers a better understanding of the domain by presenting additional information on a certain topic or indicator. This information can be presented in several media satisfying individual needs and preferences. Primarily this facility enhances understanding of domain knowledge and of how the indicators affect each other, but also supports the user in gaining control and initiative. The information provided by the 'Effects' button also helps to diminish difficulties generated by the complexity of the domain.

Problems concerning the misunderstanding of the system's strategic reasoning process can be mitigated by providing explanations on several levels, each demonstrating a different perspective. The form to be filled in, the whyand how-explanations and the presentations of rules are three examples of features which simplify understanding of the reasoning process. An alternative is the inclusion of a metalevel. When moving from rule premises to rule groups an alternative perspective is offered on the reasoning. The understanding of the system's reasoning is also supported by the division of the presentation of 
results in intermediate results and a final conclusion. Also information concerning vital indicators contributes to a further understanding of the domain.

\section{Does the structure of the system offer solutions?}

Viewed from a different perspective the system consists of three major components: the user interface, a knowledge system and a hypermedia system. Each of these components contributes in its own way and through complementary interrelations to alleviate the problems described above. Hypermedia systems by nature offer flexibility and representation of information in several media. Individual relations between units of information, information represented with difficulty otherwise and views from different angles may be represented in these systems. The flexibility lies primarily in obtaining additional information about the system's reasoning and about the domain. Why was a question posed, how should a measurement be taken, what is the margin of error of an indicator and how does it compare to that of another indicator? Answers to these questions may make the difference between truly penetrating the overall problem or just interacting with the system. However, offering the student a large amount of flexibility and freedom may, and probably will, lead to other problems, for instance user disorientation. However, these can be overcome by for example [7] overview maps, guided tours and position indicators.

The knowledge system is a valuable component in the system. It offers the possibility to infer correct conclusions, even with partial knowledge, and in addition to evaluate the presented conclusions which is essential when penetrating a new domain. In addition the transparency of the systems reasoning may, when presented in a suitable context, offer important insights.

The implication from the above is that both knowledge and hypermedia component are useful in problem solving. The complexity of the domain is primarily attacked by the hypermedia facilities, while problems of control, initiative and overview are overcome through the interface, but also by both the hypermedia and knowledge system.

\section{FURTHER WORK}

Student need to be previously acquainted with the domain through teacher or school book. We believe that it would be advantageous for the system to include domain information, preferably implemented in a flexible structure and suited to a wider audience. In this way students may obtain information tailored to their needs and level of knowledge. This feature can be seen in the upper left corner (Basic Information) of Figure 2. The implementation of 'Effects' and 
'Supporting \& Contradictory Indicators' is at present not fully developed, but will be in the near future.

\section{REFERENCES}

1. Edman, A. and Sundling, L. (1991) An Educational Knowledge-based Expert System for Environment Protection. Proceedings of the Sixth International PEG Conference, Rapallo, Italy, pp. 124-128.

2. Edman, A., Lindman, Å., and Sundling L. (1993) Design Issues Concerning Explanations in a Educational Expert System-Case Study. Proceedings of the Seveth International PEG Conference, Edinburgh, England, pp. 108-115.

3. Clancey, W. J. (1985) Use of MYCIN's Rules for Tutoring, in Buchanan, B. G. and Shortliffe, E. H. (eds.) Rule-based Expert Systems, Addison-Wesley, p. 464.

4. Conklin, J. (1987) Hypertext: An Introduction and Survey. IEEE Computer, 20 (9) pp. 17-41.

5. Anderson J. R. (1990) Cognitive Psychology and its Implications, Third Edition. W. H. Freeman and Company, p. 178.

6. Shneiderman, B. (1986) Designing the User Interface-Strategies for Effective Human Computer Interaction. Addison-Wesley.

7. Bender, A. (1992) Alleviation of User Disorientation in Hypertext Documents. Thesis for the degree of Master of Science in Computing Science, Department of Computing Science, Uppsala University. 